\title{
Annals of Neonatology Journal
}

RESEARCH ARTICLE

Assessment of serum levels of vitamin B12 in full term neonates with indirect

\section{hyperbilirubinemia}

\section{Gamal B. Mohamed ${ }^{1}$, Reem A. Salam ${ }^{1}$, Rabie H. Mortada ${ }^{1^{*}}$}

*Correspondence: Rabie H. Mortada. Department of Pediatrics, Faculty of Medicine, Minia University, Egypt.

Email: Rabiemortada123@gmail.com

Full list of author information is available at the end of the article DOI: $10.21608 /$ ANJ.2020.69363

\section{Abstract}

Introduction: Neonatal hyperbilirubinemia is defined as a total serum bilirubin level above $5 \mathrm{mg}$ per $\mathrm{dl}$ ( $85 \mu \mathrm{mol} / \mathrm{L}$ ). Jaundice is an important problem in the first week of life. It is a cause of concern for the physician and a source of anxiety for the parents.

Objective: The aim of this work was to measure the serum levels of vitamin B12 in neonates with indirect hyperbilirubinemia and to compare them with that of apparently healthy neonates.

Subjects and Methods: This study included 80 full term neonates divided into 2 groups: Group I: 40 full term neonates with indirect hyperbilirubinemia (23 females and 17males) aged 3-7 day's admitted to neonatal unit of Minia university hospital during the period from July to December 2017. Group II: 40 apparently healthy full term neonates as controls (20 males and 20 females) of matched age and weight. Results: the vitamin B12 serum levels were significantly lower than in neonates with indirect hyperbilirubinemia than controls $(\mathrm{P}$-value $=0.001 *)$. No statistically significant difference regarding age, sex, mode of delivery, gestational age or weight between patients and controls as were present. Also No statistically significant difference regarding the hemoglobin levels, platelets count, WBCS count or neutrophils count between cases and controls.

Conclusion: Full term neonates with indirect hyperbilirubinemia had lower serum levels of vitamin B12 than healthy full term neonates.

Key words: Vitamin B12, full term, neonates, hyperbilirubinemia 


\section{Introduction}

Neonatal hyperbilirubinemia is defined as a total serum bilirubin level above $5 \mathrm{mg}$ per dl $(85$ $\mu$ mol per L). Jaundice is an important problem in the first week of life. It is a cause of concern for the physician and a source of anxiety for the parents. Nearly $60 \%$ of term newborns become visibly jaundiced in the first week of life. In most cases it is benign and no intervention is required. Approximately 5-10\% of them have clinical significant hyperbilirubinemia mandating the use of phototherapy [1].

Unconjugated hyperbilirubinemia, is the most common form of jaundice encountered by family physicians. Infants without identified risk factors rarely have total serum bilirubin levels above $12 \mathrm{mg}$ per dl (205 M mol per L). common risk factors for hyperbilirubinmia include fetalmaternal blood group incompatibility, prematurity, and a previously affected sibling, cephalohematomas, bruisng, and trauma from instrumented delivery may increase the risk for serum bilirubin elevation [2]

Vit B12 is essential for DNA synthesis and for cellular energy production. Vitamin B12 deficiency is common, mainly due to limited dietary intake of animal foods or malabsorption of the vitamin [3].

The term "vitamin B12" refers to four cobalamins (Cbl), including methyl-Cbl and
adenosyl-Cbl, the two enzyme co-factors of methionine synthase and methylmalonyl-CoA mutase, respectively. Vitamin B12 deficiency produces clinical disorders that include mainly megaloblastic anemia, peripheral and central neurological manifestations. The clinical significance of low blood B12 concentrations in the absence of manifestations of deficiency is a matter of debate. The biochemical diagnosis of the subclinical and clinical deficiency of vitamin B12 has been enriched by several parameters, including serum methylmalonic acid, homocysteine, and holo-transcobalamine, which have been evaluated over the past two decades [2]

Vitamin B12 deficiency is known to produce unconjugated hyperbilirubinaemia due to the phenomenon of ineffective erythropoiesis. The resulting hyperbilirubinaemia In the presence of pre-existing conjugation defects such as Gilbert's syndrome, vitamin B12 deficiency can produce gross unconjugated hyperbilirubinaemia [4]

Aim of the work

The aim of this work was to measure serum level of vitamin B12 in neonates with indirect hyperbilirubinemia and compare them with that of apparently healthy neonates of the same age and weight.

Subjects and Methods 
The study was approved by the local research ethics committee of the faculty of medicine, Minia University

This study included 80 full term neonates divided into 2 groups: Group I included 40 full term neonates with indirect hyperbilirubinemia (23 females and 17 males) aged 3-7 day's during the period from July to December 2017. Group II included 40 apparently healthy full term neonates as a control group (20 males and 20 females) of matched age and weight.

All neonates had informed consents from their parents and all were subjected to the following: 1-Complete history taking with stress on the onset of appearance of jaundice, gestational age, mode of delivery and history suggestive of sepsis (bad general condition, fever, poor `suckling) or suggestive obstructive jaundice (dark urine and pale stool).

2-Clinical examination was done with stress on general condition jaundice, pallor, fever, vital signs (temperature, respiratory rate, and heart rate), reflexes, weight, and systemic examination for organomegaly.

3-Laboratory investigations: Complete blood count, C-reactive protein, Total and direct bilirubin, Serum vitamin B12 level, Blood group for the neonates and their mothers and Comb's test

Inclusion criteria:
1-Age 3-7 days

2-Gestational age of 37-42 weeks

3- Birth weight from $2500 \mathrm{gm}$ to $3700 \mathrm{gm}$

4-Good general condition (good moro good suckling)

5-Laboratory investigation:

-Total bilirubin more $5 \mathrm{mg} / \mathrm{dl}$

-Direct bilirubin $<20 \%$

-CBC: normal $\mathrm{Hb}$ level, platelet count and WBCs and no other signs of infection.

- Negative CRP

Sample procedure: Four ml of venous blood were obtained from each neonate under sterile venipuncture and divided as follow: One $\mathrm{ml}$ on EDTA containing tube for performing CBC. Three ml were put on plain plastic tube, kept in the incubator for 20 minutes and centrifuged at 3000r.p.m for 10 minutes to obtain serum which was then processed for bilirubin and CRP while the remaining serum stored at $-20 \mathrm{c}$ in Eppendorf cups until processing serum vitamin B12.

A-CBC assessment: It was done by automated cell counter sysmex KX21N (Japan).

B-Serum bilirubin: It was done by automated chemical analyzer konelab 201(FILAND).

\section{Statistical analysis:}

Data were entered and analyzed by SPSS 19 . Graphics by excel. Quantitative data presented as mean and standard deviation, qualitative data presented by frequency distribution. 
Independent sample $\mathrm{t}$ test, chi-square test and Pearson correlation were used. $\mathrm{P}$ value of less than 0.05 considered as cutoff for significance.

Results: There were no statistical differences regarding age, sex, mode of delivery, gestational age or weight between patients and controls as well as the values of hemoglobin, platelets, WBCS or neutrophils counts (Tables $1 \& 2$ ).

There was a significant decrease in serum levels of vitamin B12 in patients compared to controls $\left(\mathrm{P}\right.$-value $\left.=0.001^{*}\right)($ Table $3 \&$ Figure 1$)$ while there was a significant increase in total serum bilirubin in cases compared to controls as expected (Table 3).

\section{Discussion}

Vitamin B12 (Cobalamin) is a water-soluble vitamin that is derived from animal products meat, dairy, and eggs. Intrinsic factor is a glycoprotein that is produced by parietal cells in the stomach and necessary for the absorption of B12 in the terminal ileum such as red. Once absorbed, B12 is used as a cofactor for enzymes that are involved in the synthesis of DNA, fatty acids, and myelin. As a result, a B12 deficiency can lead to hematologic and neurologic symptoms. B12 is stored in excess in the liver; however, in cases in which B12 cannot be absorbed for a prolonged period (e.g., dietary insufficiency, malabsorption, lack of intrinsic factor), hepatic stores are depleted, and deficiency occurs [5].

The importance of folate and vitamin B12 for healthy neurological development and function is unquestioned. Folate and vitamin B12 are required for biological methylation and DNA synthesis. Vitamin B12 also participates in the mitochondrial catabolism of odd-chain fatty acids and some amino acids. Inborn errors of their metabolism and severe nutritional deficiencies cause serious neurological and hematological pathology. Poor folate and vitamin B12 status is associated with increased risk of cognitive impairment, depression, Alzheimer's disease and stroke among older adults and increased risk of neural tube defects among children born to mothers with low folate status[6]

$\mathrm{NNH}$ is a frequently encountered problem. Although up to $60 \%$ of term newborns have clinical jaundice in the first week of life, few have significant underlying disease. Physiologic jaundice in healthy term newborns follows a typical pattern. All etiologies of jaundice beyond physiologic and breastfeeding or breast milk jaundice are considered pathologic. Pathologic jaundice can result from $\mathrm{ABO}$ and Rh incompatibiliy, G6PD deficiency, urinary tract infection, and hypothyroidsm. So that in our study, newborns who have an indirect 
bilirubin level above $5 \mathrm{mg} / \mathrm{dL}$ were submitted to a systemic protocol which composed of $\mathrm{ABO}$, $\mathrm{Rh}$ groups, screening for G6PD, thyroid function tests, and urinary culture[7].

Here in our study, Vitamin B12 level was analyzed for a risk factor of $\mathrm{NNH}$. We found that low Vitamin B12 level is a risk factor for developement of NNH. Vitamin B12 deficiency is a correctable cause for hemolysis. The mechanism of hemolysis in this condition is ineffective erythropoiesis, where in immature erythrocytes are lysed within the bone marrow itself, resulting in the release of excess quantities of biliverdin, which is ultimately converted to indirect bilirubin[8].

Vitamin B12 (cobalamin) is essential for folate metabolism and DNA synthesis, acting as a cofactor for key enzymatic reactions. Deoxyadenosylcobalamin, one of the coenzyme forms of cobalamin, is a cofactor for methionine synthase, the enzyme which converts homocysteine to methionine. In maternal Vitamin B12deficiency, the products homocysteine and methylmalonic acid accumulate and can be transmitted to the fetus. Raised levels of these products have been detected on newborn screening of infants with low Vitamin B12 stores [9].

Vitamin B12 deficiency in newborn, in most cases, results from a maternal deficiency.
Maternal causes of infant deficiency can be divided into deficient maternal diet or maternal pernicious anemia in a breastfed infant because infant formulas are supplemented with Vitamin B12 [10]. In view of maternal diet serum Vitamin $\mathrm{B}_{12}$ concentrations decrease during pregnancy more than can be accounted for by hemodilution. There is some evidence of increased Vitamin B12 absorption during pregnancy, with newly absorbed Vitamin B12 being more important to placental transport than maternal liver stores [11].

There is a positive relation between maternal and breast-fed infant. Vitamin B12 deficiency linked to low Vitamin $\mathrm{B}_{12}$ levels in breast milk. According to a study by Specker and colleagues 1990 [12], Vitamin $B_{12}$ milk concentrations less than $360 \mathrm{pmol} / \mathrm{L}$, approximately corresponding to a maternal serum Vitamin $B_{12}$ of less than $300 \mathrm{pmol} / \mathrm{L}$ could result in an infant who is biochemically deficient in Vitamin B12. So that low Vitamin B12 uptake may cause "notenough breast milk-jaundice" that may occur when the baby does not drink enough breast milk.

A careful review of available medical literature did yield rare previous reports of severe Vitamin B12 deficiency presenting with isolated indirect hyperbilirubinemia without concomitant significant hematologic or neurologic 
involvement. There are case reports of infant Vitamin B12 deficiency being diagnosed on newborn screening. Dasari et al, 2012[4] reported a 41-year-old Asian-Indian male who presented with recurrent episodes of jaundice since Vitamin B12 deficiency.

It has been shown that neonatal jaundice shows statistically significant association with MTHFR 677T (Methylene Tetra Hydro Folate Reductase) (homocysteine metabolism gene) mutation which result elevated homocysteine. Hypomethylation due to MTHFR 677T and folate deficiency, which are independent risk factors could also be a factor leading to predisposition for NNH. So that the role of Vitamin B12 in MTHFR 677T mutation related neonatal jaundice need to be functionally established in future prospective studies.

According to the results of this study we can recommend that: Level of serum vitamin B12 should be measured in all cases of indirect hyperbilirubinemia. Serum vitamin B12 should be measured in mothers of jaundiced neonates to assess the impact of maternal vitamin B12 deficiency on both neonatal vitamin B12 and TSB. Lastly, further studies should be done to investigate the benefits of vitamin B12 supplementation in neonates with hyperbilirubinemia.

\section{Conclusion}

We could conclude that serum levels of vitamin B12 were lower in jaundiced full term neonates than that of normal healthy neonates.

Conflict of interest: The authors declared no conflict of interest.

Author's contributions: MG and SR conceived the study. MR revised the patients' medical reports and the final manuscript. All authors revised the final draft of the manuscript.

\section{Acknowledgment:}

The author would like to thank the nurse staff of neonatal intensive care unit, Minia university hospital for children for their assistance in data collection of neonates.

\section{Author's details}

${ }^{1}$ Pediatric Department, Faculty of Medicine, Minia University, Egypt.

Date received: $27^{\text {th }}$ May 2019. Accepted $29^{\text {th }}$ July 2019. Published 28 January 2020.

\section{References}

1. Mishra S, Agarwal R, Deorari AK and Paul VK. Jaundice in the newborns. Indian Journal of Pediatrics. 2008; 75(1):157-63.

2. Meredith L. Porter. Hyperbilirubinmia in the Term Newborn American Academy of Family Physicians.2002;15; 65(4):599-7

3. O'leary, F. \& Samman, S. Vitamin B12 in health and disease. Nutrients.2010; 2: 29916. 
4. Dasari, S., Naha, K. \& Prabhu, M. An unusual cause for recurrent jaundice in an otherwise healthy male. Australas Med J.2012; 5: 541-3.

5. Ankar A. \& Bhjmji S. S. Vitamin, B12 (Cobalamin), Deficiency. StatPearls. Treasure Island (FL), 2017.:22-28

6. Selhub J, Bagley LC, Miller J, B vitamins, homocysteine, and neurocognitive function in the elderly. Am J Clin Nutr. 2000;71:614S-20S

7. Hannam S, McDonnell M, Rennie JM. Investigation of prolonged neonatal jaundice. Acta Paediatr. 2000; 89(6):69469.

8. Aslinia F, Mazza JJ, Yale SH. Megaloblastic anemia and other causes of macrocytosis. Clin Med Res. 2006; 4(3): 236-24.
9. Campbell CD, Ganesh J, Ficicioglu C. Two newborns with nutritional vitamin B12 deficiency: challenges in newborn screening for vitamin B12 deficiency. Haematologica. 2005; 90 (12 Suppl):ECR45.

10. Roumeliotis N, Dix D, Lipson A. Vitamin B (12) deficiency in infants secondary to maternal causes. CMAJ. 2012;184(14):1593

11. Luhby AL, Cooperman JM, Donnenfeld AM, Herrero JM, Teller DN, Wenig JB. Observations on transfer of vitamin B12 from mother to fetus and newborn. American Journal of Disease in Childhood. 1958; 96:532-33.

12. Specker BL, Black A, Allen L, Morrow F. Vitamin B-12: low milk concentrations are related to low serum concentrations in vegetarian women and to methylmalonic aciduria in their infants. Am J Clin Nutr. 1990 Dec;52(6):1073-6.

Citation: Mohamed G.B., Salam R.A., Mortada R.H. Assessment of serum levels of vitamin B12 in full term neonates with indirect hyperbilirubinemia. Ann Neo J. 2020; 2(1): 20- 26

Copyright: Mohamed G.B. et al., 2020. This article is an open access article distributed under the terms and conditions of the Creative Commons Attribution (CC BY) license 4). 